\title{
Uso dos dermápteros no cenário agronômico: uma análise bibliométrica sobre a
}

\section{utilização destes predadores}

\author{
Use of dermápteros in the agronomic scenario: a bibliometric analysis on the use of these predators \\ Uso de dermápteros en el escenario agronómico: un análisis bibliométrico sobre el uso de estos \\ depredadores
}

Recebido: 06/03/2021 | Revisado: 12/03/2021 | Aceito: 15/03/2021 | Publicado: 22/03/2021

\author{
Angélica da Silva Salustino \\ ORCID: https://orcid.org/0000-0002-5562-0122 \\ Universidade Federal da Paraíba, Brasil \\ E-mail: angelicasalustino@gmail.com \\ Manoel Cícero de Oliveira Filho \\ ORCID: https://orcid.org/0000-0001-9053-6586 \\ Universidade Federal da Paraíba, Brasil \\ E-mail: manoel.cicero07@hotmail.com \\ Khyson Gomes Abreu \\ ORCID: https://orcid.org/0000-0002-3439-6598 \\ Universidade Federal da Paraíba, Brasil \\ E-mail: khysonabreu@gmail.com \\ Renan Rodrigues Ferreira \\ ORCID: https://orcid.org/0000-0002-8731-287X \\ Universidade Federal da Paraíba, Brasil \\ E-mail: renan_web@hotmail.com \\ Carlos Henrique de Brito \\ ORCID: https://orcid.org/0000-0002-0195-0986 \\ Universidade Federal da Paraíba, Brasil \\ E-mail: chbritoufpb@gmail.com
}

\begin{abstract}
Resumo
Objetivou-se com esse estudo inventariar os trabalhos científicos publicados nos últimos 10 anos sobre o uso de dermápteros no cenário agronômico. Para isto, realizou-se uma revisão de literatura buscando por pesquisas científicas publicadas sobre a utilização de dermápteros no cenário agronômico. A busca por essas informações ocorreu entre os meses de dezembro de 2020 e fevereiro de 2021 nas bases de dados: Web of science, ScienceDirect, Taylor \& Francis, Springer e Scielo, utilizando como palavras-chave "Dermaptera"e "Predadores". Os critérios de inclusão dos estudos na revisão foram o idioma (artigos em inglês, português e espanhol), artigos de pesquisa, data de publicação (publicados nos últimos 10 anos) e relação com a temática estudada. Após a seleção baseada nos critérios supracitados, os estudos selecionados foram lidos na íntegra e os dados coletados foram organizados em uma planilha utilizando o Microsoft Excel®. Com base nos critérios de elegibilidade, encontrou-se um total de 1.626 estudos, destes 466 foram selecionados para leitura completa e após a leitura, apenas 65 artigos corresponderam ao tema. Destes, foi possível obter informações como: espécies de dermápteros estudadas, áreas de estudos, distribuição geográfica, anos de publicação, áreas de aplicação e espécies de pragas predadas. O levantamento enaltece a escassez de pesquisas sobre a temática,no entanto, destaca-se uma alta quantidade de estudos acerca da espécie Forficula auricularia L., 1758. A pesquisa em questão aponta o Brasil como o país que mais publicou sobre o tema e revela que o foco dos estudos fez referência a utilização desses insetos no controle biológico de pragas agrícolas.

Palavras-chave: Dermaptera; Predadores; Controle biológico.
\end{abstract}

\begin{abstract}
The objective of this study was to make an inventory of the scientific works published in the last 10 years on the use of dermopterans in the agronomic scenario. To this end, a literature review was carried out, looking for published scientific research on the use of dermapters in the agronomic scenario. The search for this information occurred between the months of December 2020 and February 2021 in the databases: Web of science, ScienceDirect, Taylor \& Francis, Springer, Scielo, using as keywords "Dermaptera", "Predators". The inclusion criteria for studies in the review were language (articles in English, Portuguese and Spanish), research articles, date of publication (published in the last 10 years) and relationship with the subject studied. After the selection based on the aforementioned criteria, the selected studies were read in full and the data collected were organized in a spreadsheet using Microsoft Excel. Based on the eligibility criteria, a total of 1,626 studies were found, of which 466 were selected for complete reading, after reading, only 65 articles corresponded to the topic. From these it was possible to obtain information such as:
\end{abstract}


studied dermopteran species, study areas, geographic distribution, years of publication, area of application and species of predated pests. The survey highlights the scarcity of research on the subject. However, the high amount of studies about the species Forficula auricularia stands out. The research in question, points to Brazil as the country that most published on the subject, and reveals that the focus of the studies made reference to the use of these insects in the biological control of agricultural pests.

Keywords: Dermaptera; Predators; Biological control.

\section{Resumen}

El objetivo de este estudio fue realizar un inventario de los trabajos científicos publicados en los últimos 10 años sobre el uso de dermápters en el escenario agronómico. Para ello, se realizó una revisión de la literatura, buscando investigaciones científicas publicadas sobre el uso de dermapters en el escenario agronómico. La búsqueda de esta información se produjo entre los meses de diciembre de 2020 y febrero de 2021 en las bases de datos: Web of science, ScienceDirect, Taylor \& Francis, Springer, Scielo, utilizando como palabras clave "Dermaptera", "Depredadores". Los criterios de inclusión de los estudios en la revisión fueron idioma (artículos en inglés, portugués y español), artículos de investigación, fecha de publicación (publicados en los últimos 10 años) y relación con el tema estudiado. Luego de la selección en base a los criterios antes mencionados, los estudios seleccionados fueron leídos íntegramente y los datos recolectados fueron organizados en una hoja de cálculo utilizando Microsoft Excel. Con base en los criterios de elegibilidad, se encontraron un total de 1.626 estudios, de los cuales se seleccionaron 466 para lectura completa, luego de la lectura, solo 65 artículos correspondieron al tema. De estos se pudo obtener información como: especies de dermopteran estudiadas, áreas de estudio, distribución geográfica, años de publicación, área de aplicación y especies de plagas depredadoras. La encuesta destaca la escasez de investigaciones sobre el tema. Sin embargo, destaca el elevado número de estudios sobre la especie Forficula auricularia. La investigación en cuestión, apunta a Brasil como el país que más publicó sobre el tema, y revela que el foco de los estudios hizo referencia al uso de estos insectos en el control biológico de plagas agrícolas.

Palabras clave: Dermaptera; Depredadores; Control biológico.

\section{Introdução}

Dermaptera (derma=pele, ptera=asa, em referência a textura das asas anteriores) é uma das ordens da classe Insecta que contém os animais conhecidos popularmente como tesourinhas ou lacrainhas. Eles são insetos hemimetábolos, terrestres, de corpo alongado, que podem medir de 3 a 85 milímetros de comprimento. Apresentam uma coloração uniforme, variando em tons de preto, marrom e amarelo, sendo facilmente reconhecidas pelos cercos em forma de pinças no final do abdome. Algumas espécies são capazes de voar, enquanto outras nem sequer apresentam asas, mas todas elas apresentam tigmotaxia, preferindo sempre estar em contato com alguma superfície (Rafael et al., 2012).

São encontradas por todo o mundo, com exceção da Antártida, mas apresentam maior diversidade de espécies em regiões intertropicais, justamente por fatores biogeográficos associados a diversificação de espécies nessa região (Haas, 2019). Em sua maioria, possuem hábito noturno e durante o dia costumam se esconder da luz, seja na serrapilheira, em frestas de troncos ou na bainha de folhas. Nesse último caso, apresenta significativa relevância econômica por atuar como predador natural de algumas pragas de culturas, ou no caso de algumas outras espécies, serem as próprias pragas, se alimentando de brotos e de inflorescências, comprometendo o desenvolvimento dos cultivos, assim como a qualidade do seu produto final (Triplehorn \& Johnson, 2015).

Por apresentarem muitas espécies onívoras, acabam sendo, também, excelentes predadores, sobretudo sobre insetospraga, se alimentando desde ovos a formas jovens. De acordo com Silva e Batista (2018), dermápteros foram observados predando pragas em várias culturas de relevância como maçã, ervilha, alfafa, cana-de-açúcar, citrus, algodão, sorgo e milho, sendo esses insetos de várias ordens, tais como Coleoptera, Lepidoptera e Hemiptera.

Os dermápteros podem ocorrer tanto espontaneamente nas lavouras como também serem introduzidos a fim de substituir os agrotóxicos. Com isso, os estudos de interesse agronômico com as tesourinhas acabam girando em torno do seu potencial predatório (Silva, Batista \& Brito, 2009), da seletividade desses insetos a outras substâncias que podem ser utilizadas em conjunto (Campos et al., 2011), assim como o comportamento, a biologia e as metodologias de criação desses insetos em 
laboratório (Greer et al., 2020; Miller \& Zing, 2012; Alves, 2014), uma vez que se necessita de uma grande quantidade de tesourinhas para a realização de bioensaios e outros experimentos.

Diante das muitas possibilidades de utilização dos dermápteros no âmbito agronômico, e do seu alto potencial no controle de pragas, as pesquisas nessa temática ainda são bastante escassas, sendo um campo de alto potencial exploratório em estudos futuros. Portanto, é de suma importância a realização de pesquisas dessa natureza, uma vez que reunirá informações atuais do campo científico sobre a utilização de dermápteros no meio agrícola. Assim, buscou-se nessa pesquisa, inventariar os trabalhos científicos publicados nos últimos 10 anos sobre o uso de dermápteros no cenário agronômico.

\section{Metodologia}

No primeiro momento foi realizada a revisão bibliográfica, baseada na busca por pesquisas científicas publicadas sobre insetos da ordem Dermaptera utilizados no cenário agronômico. A obtenção dessas informações foi realizada entre os meses de dezembro de 2020 a fevereiro de 2021 nas seguintes bases de dados: Web of Science, ScienceDirect, Taylor \& Francis, Springer, Scopus.

Artigos em inglês, português e espanhol publicados nos últimos 10 anos e que estavam disponíveis na íntegra, relacionados ao uso de dermápteros na agricultura, foi um dos critérios utilizados para selecionar os trabalhos. Para a realização da busca foram utilizadas as seguintes palavras-chave: "Dermaptera", "Predadores", pesquisados tanto em português, quanto em inglês. Os operadores booleanos utilizados foram AND e OR.

A busca foi realizada nas bases de dados anteriormente citadas através da combinação das palavras-chave, sendo a última data de acesso 18 fevereiro de 2020, e os resultados foram importados para uma biblioteca do Mendeley Gerente de referência, removendo-se as duplicatas. Foram tidos como inadequados para compor o presente estudo os trabalhos no formato de monografia, dissertação, tese e projeto de pesquisa.

Os registros restantes foram recuperados em texto completo e inspecionados em detalhes para inclusão no estudo, inicialmente por seus títulos, por seguinte leitura dos resumos e aqueles que atendiam aos critérios de inclusão da pesquisa foram lidos na íntegra. Para organização dos dados coletados nos artigos foi elaborada uma planilha utilizando o Microsoft Excel ${ }^{\circledR}$ contendo as seguintes informações: título do artigo, nome da base de dados, nome do periódico, ano de publicação, área de estudo, espécie predada, local da realização da pesquisa, distribuição geográfica e espécie estudada.

\section{Resultados}

Com base nos critérios de elegibilidade, de 1.626 estudos encontrados na busca nas bases de dados, apenas 466 artigos foram selecionados para leitura completa. Após a leitura, 65 artigos foram selecionados para esta revisão (Figura 1).

Figura1. Fluxograma baseado no modelo PRISMA com os resultados da seleção dos artigos.

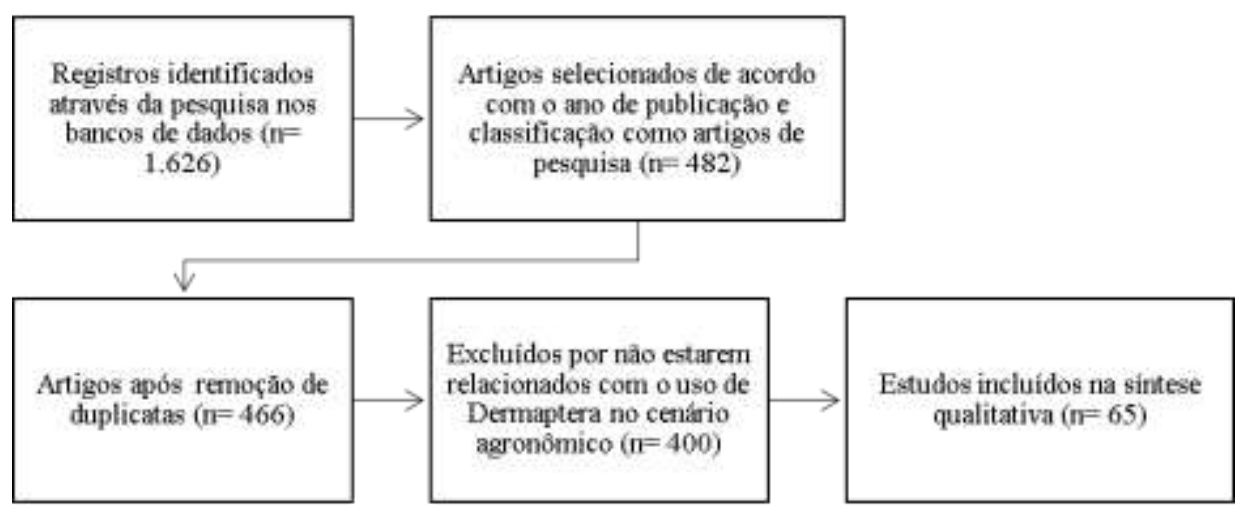




\subsection{Espécies estudadas}

Fonte: Acervo da pesquisa.

Foram encontrados 37 trabalhos com a espécie Forficula auricularia L., 1758 (Figura 2), também conhecida como tesourinha europeia, e dentre esses, 26 trabalhos foram desenvolvidos em algum país europeu, sendo a espécie mais citada.

Figura 2. Espécies de dermápteros estudadas no cenário agronômico no período de 2010 a 2020.

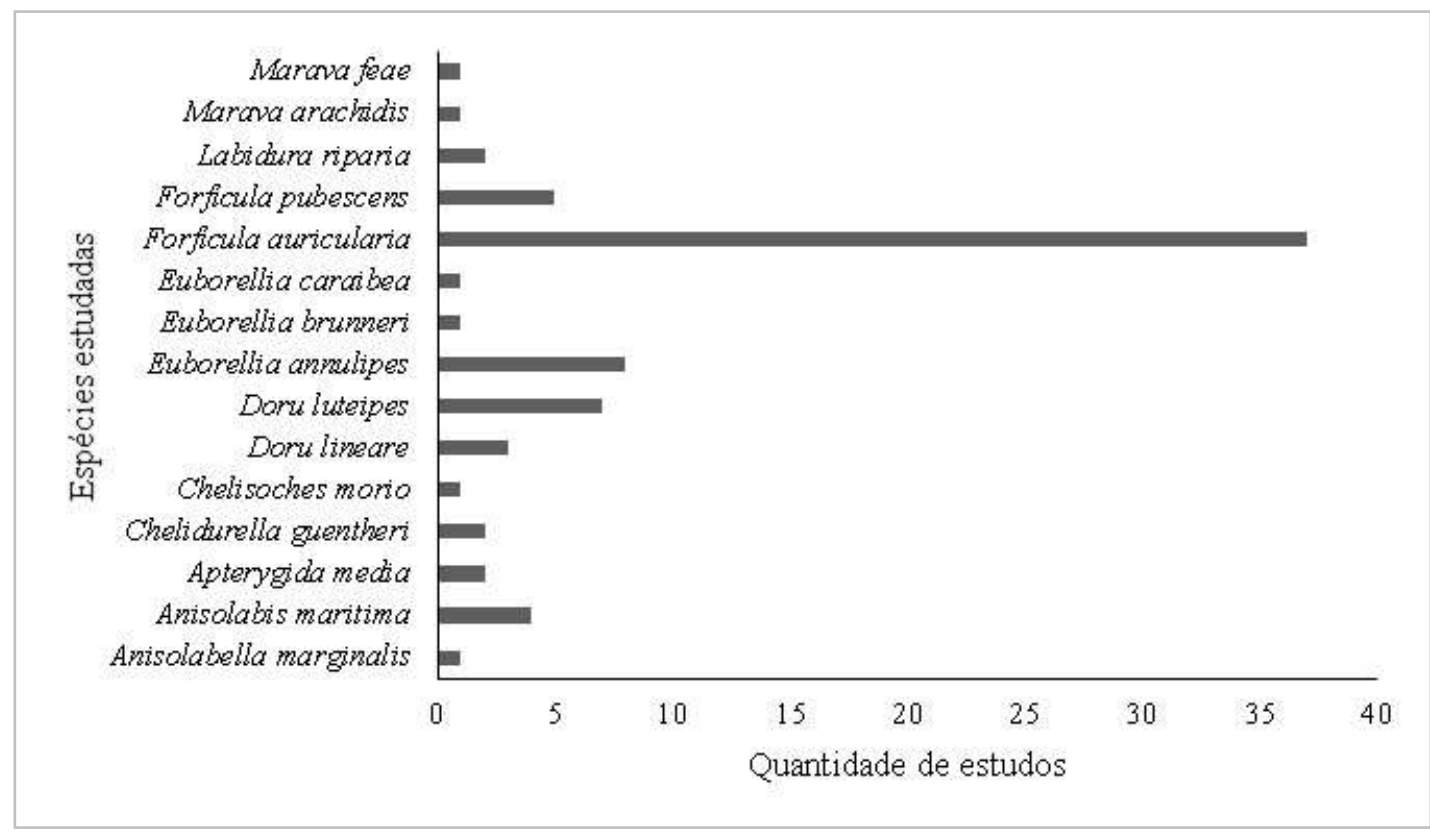

Fonte: Acervo da pesquisa.

\section{2 Áreas de estudos}

Quanto as áreas de estudos (Figura 3), observou-se que o maior percentual dos artigos encontrados sobre o uso de dermápteros no cenário agronômico fazem referência à sua utilização dentro da área de controle biológico, seguido de pesquisas referentes a biologia desses predadores e também estudos na área de seletividade desses organismos a produtos químicos. Em menor número, foram encontrados artigos abordando a ecologia, o manejo destes como pragas e artigos sobre a sistemática filogenética. 
Figura 3. Áreas de estudos envolvendo o uso de dermápteros no cenário agronômico durante o período de 2010 a 2020 (\%).

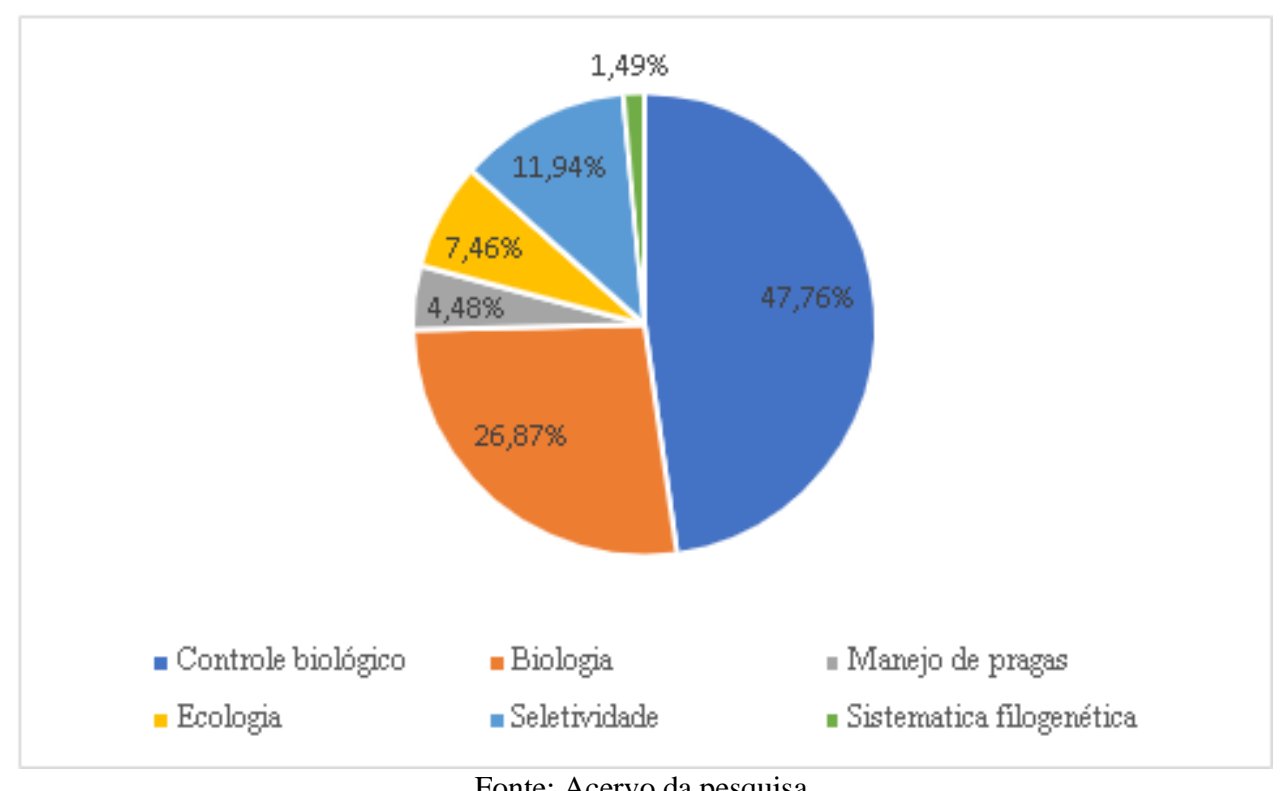

\subsection{Distribuição geográfica}

De acordo com a distribuição geográfica da pesquisa sobre a utilização de dermápteros no cenário agronômico, é possível observar na Figura 4, que a concentração dos estudos ocorreu no Brasil e na França, seguidos dos Estados Unidos da América (EUA) e Austrália ambos com o mesmo número de artigos encontrados, prosseguindo com Espanha e Alemanha, e além desses, outros em menor número.

Figura 4. Distribuição geográfica da pesquisa sobre o uso de dermápteros no cenário agronômico durante o período de 2010 a 2020.

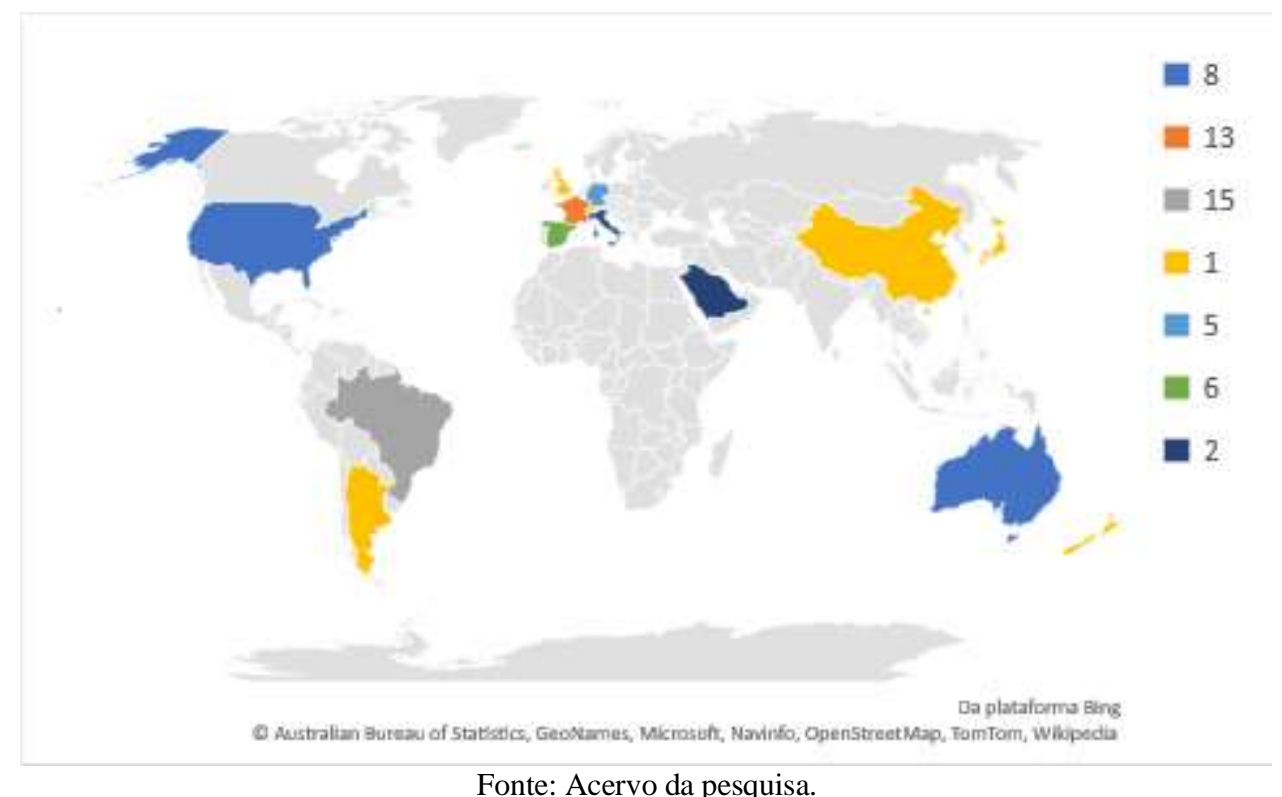

Fonte: Acervo da pesquisa. 


\subsection{Anos de publicação}

A tendência temporal observada na Figura 5 apresentou um comportamento irregular para a distribuição das publicações sobre a utilização de dermápteros no cenário agronômico. No entanto, foi observado um aumento crescente entre os anos de 2019 e 2020, onde juntos somam 33,3\% dos 66 artigos encontrados sobre a temática abordada.

Figura 5. Tendência temporal da pesquisa sobre o uso de dermápteros no cenário agronômico durante o período de 2010 a 2020.

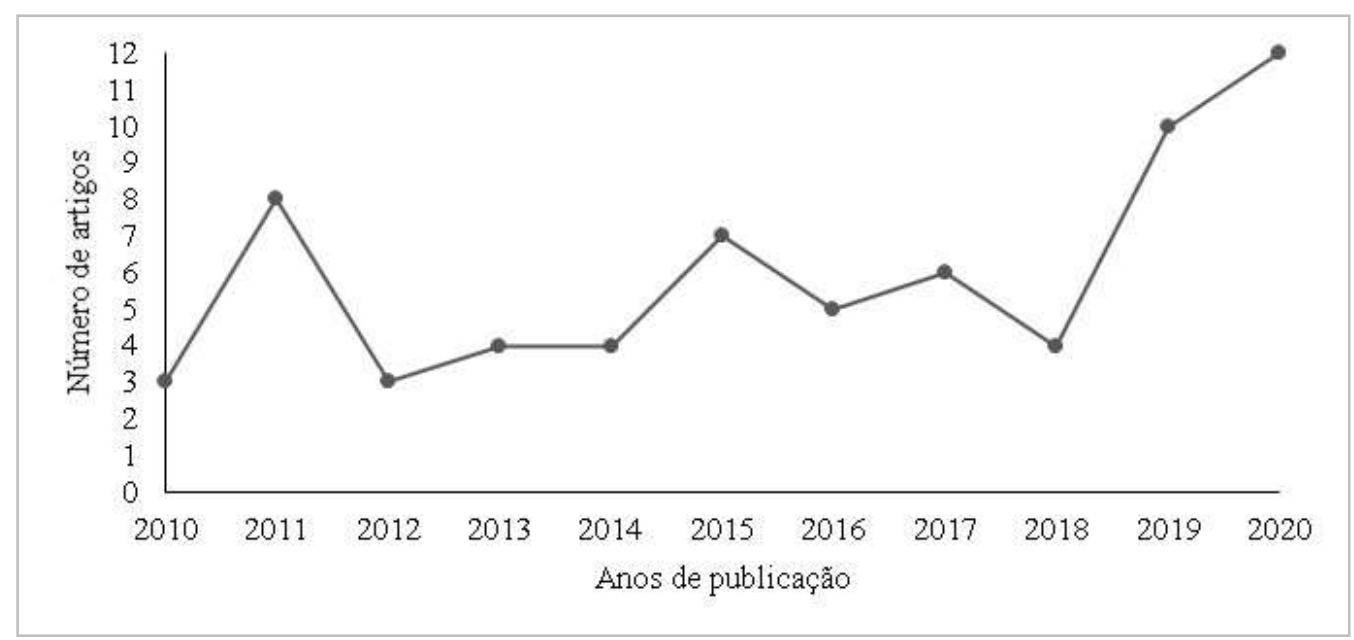

Fonte: Acervo da pesquisa.

\subsection{Locais de realização da pesquisa}

Quanto ao levantamento dos locais de estudos, conforme a Figura 6, observa-se uma maior proporção em laboratório com cerca de $64 \%$, seguido do campo com cerca de $33 \%$ e por fim laboratório/campo com $3 \%$.

Figura 6. Levantamento dos locais de realização da pesquisa sobre o uso de dermápteros no cenário agronômico durante o período de 2010 a 2020.

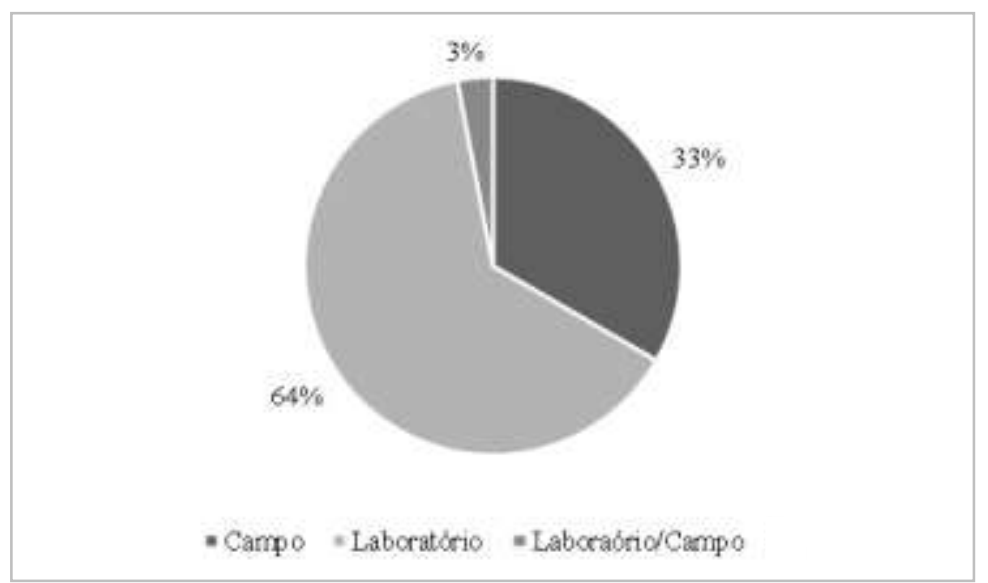

Fonte: Acervo da pesquisa.

\subsection{Espécies predadas}

Ao observar a Figura 7, nota-se que dentre os insetos-praga predados por diferentes espécies de tesourinhas, destacase a presença do pulgão-rosado-da-maçã (Dysaphis plantaginea), onde pode ser constatado um maior número de estudos com 
esse inseto no período avaliado. No entanto, outras pragas também aparecerem em número expressivo como Eriosoma lanigerum e Plutella xylostella.

De maneira geral, levando em consideração o intervalo de tempo analisado, é possível observar que as pesquisas tiveram um enfoque maior sobre o uso das tesourinhas no controle de insetos da ordem Hemiptera, como pulgões e cochonilhas e também sobre insetos da ordem Lepidoptera como as borboletas e mariposas, e menos estudos sobre insetos da ordem Diptera, Coleoptera e Hymenoptera.

Figura. 7. Levantamento dos insetos-praga, predados por diferentes espécies de tesourinha durante o período de 2010 a 2020.

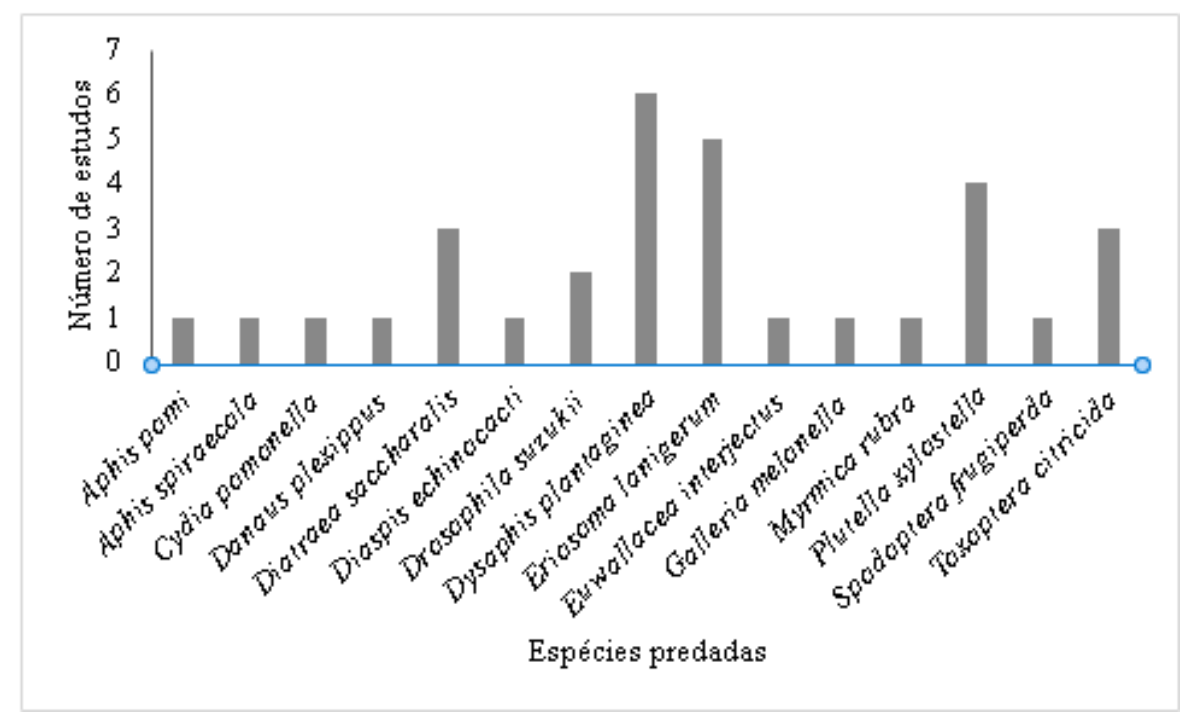

Fonte: Acervo da pesquisa.

\section{Discussão}

\subsection{Espécies estudadas}

Conforme mencionado por Rafael et al. (2012), no mundo são conhecidas cerca de 2.200 espécies de dermápteros, com um número estimado de 3.500 espécies. No entanto, de acordo com os artigos levantados nesse trabalho, levando em consideração o potencial predatório sobre pragas, apenas 15 espécies no mundo todo foram estudadas. Dessas, a espécie $F$. auricularia apresentou uma quantidade significativa de pesquisas, o que pode ser explicado pelo grande impacto que esta apresenta, tanto negativa como positivamente, sobretudo na Europa.

De acordo com o Centro de Biociência Agrícola Internacional (CABI) (2019), a F. auricularia apresenta grande potencial predatório, sendo um excelente agente para o controle de pragas como pulgões e cochonilhas. Esse fator somado a sua ampla distribuição pelo mundo faz com que a tesourinha europeia seja a mais estudada nos últimos 10 anos, sobretudo na Europa.

As demais espécies não apresentaram quantidade expressiva de pesquisas realizadas, alcançando no máximo oito pesquisas no mundo todo entre 2010 e 2020. Alguns pontos podem justificar tal escassez, a exemplo da baixa relevância dessas espécies em lavouras de forte impacto econômico, falta de conhecimento acerca de sua importância e a dificuldade de estabelecer uma criação em laboratório para uso em pesquisas. 


\section{2 Áreas de estudos}

O maior percentual de pesquisas na área de controle biológico se dá pela importância desses predadores no controle de pragas de culturas agrícolas, o que desperta o interesse de estudos voltados para essa área, buscando identificar o potencial predatório das espécies e a suas relações tróficas com as presas (Sousa \& Matta, 2019).

Já os estudos relacionados à biologia desses predadores também possuem relevância dentro do cenário agronômico, uma vez que através de pesquisas nesse sentido, é possível compreender melhor os aspectos biológicos dos dermápteros como o número de instares, viabilidade ninfal, peso e tamanho ao se alimentarem de diferentes predadores ou dietas artificiais (Alves et al., 2019; Silva et al., 2019) ou ainda a adaptação desses organismos a diferentes faixas de temperatura, o que possibilita a sua criação massal em condições controladas para posterior liberação em campo ou para a realização de novos estudos (Pasini et al., 2010).

As pesquisas na área de seletividade crescem no decorrer dos anos devido a necessidade da conservação do equilíbrio ecológico. Dessa forma, a utilização de produtos controladores de pragas que não afetem os predadores é de extrema importância para a manutenção da biodiversidade no meio ambiente, visto que o produto estará controlando o inseto-praga sem causar danos nos seus predadores naturais, o que contribui para um menor gasto com produtos comerciais (Redoan et al 2012).

\subsection{Distribuição geográfica}

A concentração de estudos em determinados países deve estar relacionada com a ineficiência do uso de defensivos químicos em determinados manejos, como também da necessidade de evitá-los, principalmente em cultivos de cana-de-açúcar e brássicas no Brasil e de maçã na França, tendo em vista que, a maioria dos estudos utilizando dermápteros nestes países fazem referência ao controle biológico de pragas dessas culturas.

A ineficácia dos produtos químicos é um ponto bem relatado no Brasil para a cultura da cana-de-açúcar, devido ao desenvolvimento das larvas no interior dos colmos (Nunes et al., 2019). Esse fato colabora para o aumento na busca por inimigos naturais e consequentemente a busca por estudos que comprovem eficiência bem-sucedida desses predadores.

Outro ponto que corrobora para estudos utilizando dermápteros no cenário agrícola é o crescente interesse na promoção de métodos alternativos de controle como a utilização de inimigos naturais, o que evita efeitos colaterais adversos (Le Navenant et al., 2021).

\subsection{Anos de publicação}

Foi observado aumento na quantidade de artigos nessa temática no final da década analisada, mais precisamente nos anos de 2019 e 2020. Isso pode estar relacionado a popularização dos métodos alternativos de controle de insetos-praga, que vêm forte e gradativamente substituindo o uso de compostos químicos e sintéticos nos últimos anos.

De uma forma geral, foi verificado uma pequena quantidade de trabalhos com dermápteros na década em questão e isso pode ser justificado pela alta variedade de opções de espécies predadoras com potencial de serem utilizadas no controle biológico de pragas. Visto que, segundo Parra et al. (2002), existem 22 ordens de predadores e cinco de parasitoides, fazendo do controle biológico uma área extremamente vasta para estudo, podendo ser essa uma das causas de haver relativamente poucos trabalhos com dermápteros nessa área.

\section{5 Área de aplicação da pesquisa}

O registro de uma maior quantidade de pesquisas em laboratório deve estar relacionado a facilidade da criação destes insetos em ambientes controlados (Salustino et al., 2021). Outros pontos positivos podem ser destacados como criação em massa destes insetos, fornecimento de dietas artificiais, disponibilidade de equipamentos, dentre outros fatores. Tendo em vista 
a importância de buscar resultados preliminares de interesse biológico e agronômico para ser aplicados em campo, as pesquisas em laboratórios se tornam um fator de extrema importância.

\subsection{Espécies predadas}

Dentro do período analisado houve um maior número de pesquisas avaliando a interação entre as tesourinhas e o inseto-praga Dysaphis plantaginea, conhecido vulgarmente como o pulgão- da-maçã-rosada.

A alta voracidade demonstrada pelas espécies de predadores as credenciam como agentes afidófagos promissores para uso em programas de controle biológico (Dib et al., 2020). No entanto ainda são necessárias mais pesquisas em condições de campo como ressalta Dib et al (2016). As pesquisas analisadas também demonstraram trabalhos em que as tesourinhas suprimiram os pulgões lanígero da maçã (Eriosoma lanigerum) sem causar danos no fruto (Happe et al., 2018).

Alguns estudos visavam compreender melhor a relação predador-presa, como o de Nunes et al. (2019), que demonstrou em sua pesquisa que fêmeas adultas de E. annulipes são predadoras vorazes de larvas e pupas de P. xylostella, apresentando preferência por larvas.

Orpet et al. (2019) destaca que as tesourinhas possuem uma alta voracidade, no entanto, são difíceis de serem observadas em campo e recorrentemente são interpretadas como pragas ao invés de inimigos naturais, e devido a isso podem ter os seus benefícios subestimados na agricultura.

\section{Conclusão}

A listagem de artigos sobre a utilização de dermápteros no cenário agronômico deixa clara a escassez de pesquisas sobre essa temática nos últimos 10 anos. No entanto, destaca-se a alta quantidade de estudos acerca da espécie Forficula auricularia L., 1758. O levantamento, aponta o Brasil como o país que mais publicou sobre o tema, e revela que o foco dos estudos é referente a utilização desses insetos no controle biológico de pragas agrícolas.

Esse tipo de pesquisa traz uma grande contribuição para a ciência ao reunir uma série de informações que facilitarão futuras pesquisas para a comunidade científica que se dedica a estudar esses animais.

\section{Referências}

Alves, P. R. R. (2014). Desenvolvimento e reprodução de Pygidicrana v-nigrum Serville, 1831 (Dermaptera: Pygidicranidae) alimentada com dieta artificial. Trabalho de Conclusão de Curso apresentado à Universidade Federal da Paraíba.

Alves, P. R. R., de Oliveria, R., de Oliveira Barbosa, V., de Souza, M. D. S., \& Batista, J. D. L. (2019). Desenvolvimento e reprodução de Pygidicrana vnigrum (Dermaptera: Pygidicranidae) alimentada com dieta artificial. PesquisAgro, 2 (1), 24-33.

Campos, M. R., Picanço, M. C., Martins, J. C., Tomaz, A. C., \& Guedes, R. N. C. (2011). Insecticide selectivity and behavioral response of the earwig Doru luteipes. Crop Protection, 30(12), 1535-1540.

Centre for Agriculture and Bioscience International (2019). Forficula auricularia (European earwig). Invasive Species Compendium. <https://www.cabi.org/isc/datasheet/24345>.

Dib, H., Jamont, M., Sauphanor, B., \& Capowiez, Y. (2016). Individual and combined effects of the generalist Forficula auricularia and the specialist E pisyrphus balteatus on Dysaphis plantaginea - are two predators better than one? Entomologia Experimentalis et Applicata, 161(1), 1-10.

Dib, H., Siegwart, M., Delattre, T., Perrin, M., \& Lavigne, C. (2020). Does combining Forficula auricularia L. (Dermaptera: Forfic ulidae) with Harmonia axyridis Pallas (Coleoptera: Coccinellidae) enhance predation of rosy apple aphid, Dysaphis plantaginea Passerini (Hemiptera: Aphididae)? Biological Control, 151, 104394.

Greer, J. A., Swei, A., Vredenburg, V. T., \& Zink, A. G. (2020). Parental care alters the egg microbiome of maritime earwigs. Microbial Ecology, 80(4), 920934.

Haas, F. (2019). The Earwig Research Center (ERC). The site on earwig biology. <http://www.earwigs-online.de>.

Happe, A. K., Roquer-Beni, L., Bosch, J., Alins, G., \& Mody, K. (2018). Earwigs and woolly apple aphids in integrated and organic apple orchards: responses of a generalist predator and a pest prey to local and landscape factors. Agriculture, Ecosystems \& Environment, $268,44-51$. 
Research, Society and Development, v. 10, n. 3, e46110313611, 2021

(CC BY 4.0) | ISSN 2525-3409 | DOI: http://dx.doi.org/10.33448/rsd-v10i3.13611

Le Navenant, A., Brouchoud, C., Capowiez, Y., Rault, M., \& Suchail, S. (2021). How lasting are the effects of pesticides on earwigs? A study based on energy metabolism, body weight and morphometry in two generations of Forficula auricularia from apple orchards. Science of The Total Environment, 758, 143604.

Miller, J. S., \& Zink, A. G. (2012). Parental care trade-offs and the role of filial cannibalism in the maritime earwig, Anisolabis maritima. Animal behaviour, $83(6), 1387-1394$

Nunes, G. D. S., Dantas, T. A. V., de Souza, M. D. S., do Nascimento, I. N., de Luna Batista, J., \& Malaquias, J. B. (2019). Life stage and population density of Plutella xylostella affect the predation behavior of Euborellia annulipes. Entomologia Experimentalis et Applicata, 167(6), 544-552.

Nunes, G. S., Ramalho, D. G., Dos Santos, N. A., Truzi, C. C., Vieira, N. F., Cardoso, C. P., \& De Bortoli, S. A. (2019). Parasitism-mediated interactions between the ring-legged earwig and sugarcane borer larvae. Neotropical entomology, 48(6), 919-926.

Orpet, R. J., Goldberger, J. R., Crowder, D. W., \& Jones, V. P. (2019). Field evidence and grower perceptions on the roles of an omnivore, European earwig, in apple orchards. Biological Control, 132, 189-198.

Parra, J. R. P. (2002). Controle biológico no Brasil: parasitóides e predadores. Editora Manole Ltda.

Pasini, A., Parra, J. R. P., Nava, D. E., \& Butnariu, A. R. (2010). Exigências térmicas de Doru lineare Eschs. e Doru luteipes Scudder em laboratório. Ciência Rural, 40(7), 1562-1568.

Rafael, J. A., Melo, G. A. R., Carvalho, C. J. B. D., Casari, S. A., \& Constantino, R. (2012). Insetos do Brasil: diversidade e taxonomia.

Redoan, A., Carvalho, G., Cruz, I., Figueiredo, M., \& da Silva, R. B. (2012). Seletividade de inseticidas utilizados no controle de Spodoptera frugiperda (JE Smith)(Lepidoptera: Noctuidae) para ovos e ninfas de doru luteipes (Scudder)(Dermaptera: forficulidae). Embrapa Milho e Sorgo-Artigo em periódico indexado (ALICE).

Salustino, A. S., Celedônio, W. F., de Oliveira Filho, M. C., de Melo, D. S., da Silva, J. J., \& de Brito, C. H. (2021). Biological control of fruit flies: bibliometric analysis on the main biocontrol agents. Research, Society and Development, 10(1), e22510111245-e22510111245.

Silva, A. B., Batista, J. L. (c2018). Tesourinhas: pequenas e de grande eficiência. Revista Cultivar. <https://www.grupocultivar.com.br/artigos/tesourinhaspequenas-e-de-grande-eficiencia>.

Silva, A. B. D., Batista, J. D. L., \& Brito, C. H. D. (2009). Capacidade predatória de Euborellia annulipes (Lucas, 1847) sobre Spodoptera frugiperda (Smith, 1797). Acta Scientiarum. Agronomy, 31(1), 7-11.

Silva, W. A. O., de Medeiros, M. D. G., Wanderley, A., de Souza Dias, U., \& Viana, P. H. F. (2019). Comportamento predat $\tilde{A}^{3}$ rio de Euborellia annulipes em diferentes fontes de alimento. Caderno Verde de Agroecologia e Desenvolvimento Sustentável, 9(7), 6946.

Sousa, J. M. A., \& da Matta, D. H. (2019). Controle biológico conservativo: Plantas herbáceas e a diversidade e abundância de dermápteros em algodoeiro colorido. EntomoBrasilis, 12(3), 132-140.

Triplehorn, C. A., Johnson, N. F. (2015). Estudo dos insetos. (2a ed.), Cengage Learning. 